\title{
SURFACE SNOW DENSITY OF EAST ANTARCTICA DERIVED FROM IN-SITU OBSERVATIONS
}

\author{
Y.Tian ${ }^{1,2}$, S. Zhang ${ }^{1,2}$, W. Du ${ }^{1,2}$, J. Chen ${ }^{1,2}$, H. Xie ${ }^{1,2}$, X. Tong ${ }^{1,2}$, R. Li $^{1,2, *}$ \\ ${ }^{1}$ College of Surveying and Geo-Informatics, Tongji University, 1239 Siping Road, Shanghai, China \\ ${ }^{2}$ Center for Spatial Information Science and Sustainable Development, Tongji University, 1239 Siping Road, Shanghai, China
}

Commission III, WG III/9

KEY WORDS: Surface snow density, Matrix completion, Empirical Orthogonal Function, Kriging

\begin{abstract}
:
Models based on physical principles or semi-empirical parameterizations have used to compute the firn density, which is essential for the study of surface processes in the Antarctic ice sheet. However, parameterization of surface snow density is often challenged by the description of detailed local characterization. In this study we propose to generate a surface density map for East Antarctica from all the filed observations that are available. Considering that the observations are non-uniformly distributed around East Antarctica, obtained by different methods, and temporally inhomogeneous, the field observations are used to establish an initial density map with a grid size of $30 \times 30 \mathrm{~km}^{2}$ in which the observations are averaged at a temporal scale of five years. We then construct an observation matrix with its columns as the map grids and rows as the temporal scale. If a site has an unknown density value for a period, we will set it to 0 in the matrix. In order to construct the main spatial and temple information of surface snow density matrix we adopt Empirical Orthogonal Function (EOF) method to decompose the observation matrix and only take first several lower-order modes, because these modes already contain most information of the observation matrix. However, there are a lot of zeros in the matrix and we solve it by using matrix completion algorithm, and then we derive the time series of surface snow density at each observation site. Finally, we can obtain the surface snow density by multiplying the modes interpolated by kriging with the corresponding amplitude of the modes. Comparative analysis have done between our surface snow density map and model results. The above details will be introduced in the paper.
\end{abstract}

\section{INTRODUCTION}

Firn density of Antarctica can fluctuate spatially and temporally. The errors will be introduced by density value when converting volume change to mass change (Arthern et al., 2010). Furthermore, changes in grain size, structure, snow particles and properties occur during the process of snow densification which is of fundamental important in many aspects of glaciology, including the climatic signals contained in deep ice cores. (Sugiyama et al., 2012). Driven by these factors, nearsurface snow density and the process of firn densification have been studied by observations derived from field measuring or by theoretical and numerical methods, such as numerous snow densification models (Sugiyama et al., 2012).

At present, mass balance estimates of the Antarctic Ice Sheet are mainly derived from Altimetry, Gravimetry and Input-Output (IOM) methods. High-precise estimate of elevation change can be achieved by satellite altimetry measurements, which makes altimetry method thought to be more precise than other two methods. However, the determination of the snow density introduces errors to the conversion from volume to mass change (Shepherd et al., 2012). Previous estimates of the ice-mass change from the measured elevation change have followed diverse criteria. Some authors adopted an effective density ranging from 350 to $917 \mathrm{~kg} / \mathrm{m}^{3}$. Some apply firn correction models based physical principles or semi-empirical parameterizations (Gunter et al., 2009; Rignot et al, 2011; Shepherd et al., 2012; Zwally, 2015). So far, the biggest uncertainty from volume to mass change is density, according to
Shepherd et al. (2012), the error introduced by selected density for the mass balance estimated by altimetry can be up to $30 \%$. The firn density leads to more uncertainty for East Antarctica mass estimate.

The snow density profile is usually measured through following four methods. Traditional gravimetric method as the earliest way for Antarctic snow density measurement mainly aims to measure mass and volume (Hawley et al., 2008). According to the study, the resolution and accuracy depend on the sample size (Hawley et al., 2008). Comparing to the means by using a sampler (generally a rectangular sampler with a standard volume of $500 \mathrm{~cm}^{3}$ ) and a weight-balance, samples taken from ice cores can get deeper layer density values and obtained density generally is affected by the extracted core quality determined by diameter, length and weighted pieces of the core. During drilling, the mixing of snow layers will introduce errors, therefore, it is better to determine the snow density of the upper layer by using shallow pit measurements. In the neutronscattering probe (NP) method, the density profiles are obtained by counting the rate of neutron returning to the detector and are largely affected by the diameter of the borehole (Hawley et al., 2008). Morris et al. (2008) pointed out that the $10 \%$ error of the borehole diameter in the derived density will be of the order 8$10 \%$. In the dielectric profiling (DEP) method, density profiles are inferred by measuring conductivity and permittivity, and generally are subjected to core diameter effects (Hawley et al., 2008). Hawley and others (2008) indicated that errors occur in low-density sections can up to $15 \%$ and $10-13 \%$ for highdensity sections. In the core optical stratigraphy (COS) method,

* Corresponding author - rli@tongji.edu.cn 
density profiles are derived from extracting a similar core optical stratigraphy by use of digital photography to image core profiles and fine-scaled features are well captured by it (Hawley et al., 2008). NP, DEP and COS methods all provide tremendous improvements to traditional gravimetric method for density measurement with increased spatial resolution and accuracy. However, the application of these methods hasn't yet spread and only a few observations exist. Due to the vertical density profiles are scarce over the entire Antarctica, we get the near-surface snow density map from all field observations that are available.

In this paper, we present a near-surface snow density map of East Antarctica from in-situ observations by using Empirical Orthogonal Function (EOF) to extract the most information of observations that contain time series of density.

\section{DATA AND METHOD}

\subsection{Density Observations}

\subsubsection{Data Collection}

We collected snow density data from all field observations that are available, including ice cores and snow pits. There are 2239 observations in total and span from 1957 to 2015 (Fig.1). This dataset contains 28 traverse routes and 1431 observation sites with only one density value at a time.

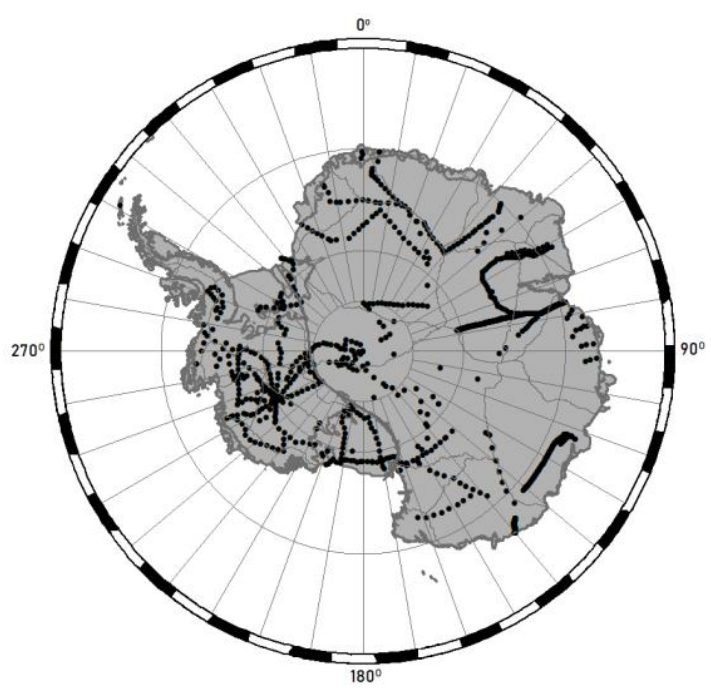

Figure 1. Locations of 2239 in-situ observations used in the map of surface snow density

\subsubsection{Data Pre-processing}

Near-surface densification has been studied by measuring the vertical profiles of firn cores and shallow pits (Sugiyama et al., 2012). These studies have found that the density changes with the depth and finally reaches the density of the ice. Considering that the depths of measured densities are different and the observations are non-uniformly distributed around Antarctica, following strategies had been taken to get neat data.

\subsubsection{Unify the Depth of Density Values}

We fit depth-density observations to get the average of observed density from 0-to2-meter depth for each density profile when needed. If the number of observations in a profile from 0-to3meter depth is more than 5 , we use a linear fit and then get the 0 -to2-meter average density. If the number is smaller than 5 and there are observations from 0 to 1 meter or 2 meters, we will directly average the observations from 0 -to2-meter depth. Otherwise, we average the density observations from 0-to1meter depth or use the exponential function model in Takahashi et al. (2007) to fit the profile and average the observations from 0 -to2-meter depth. However, initial layers' depths of the two sites on the ITASE traverse route beyond 2 meters, they should have been rejected but due to the important spatial location, we accept them by fitting and then averaging the observations from 0-to3-meter depth.

\subsubsection{Smooth the Observations}

Next, removing the observations that beyond triple root mean square error for each traverse route and the density values were smoothed by a $30 \mathrm{~km}$ running average to remove spatial and measurement noise for the traverse route that haven't done before. But the $150 \mathrm{~km}$ line measured by France locates on coastal area and has fast-changing densities, so we use segmented running average for it to retain the changeable highdensity information. After that, all densities are averaged inside a $30 \mathrm{~km}$ x $30 \mathrm{~km}$ cell at each year and also averaged about every five years at each observation site.

\subsubsection{Construct Observation Matrix}

Last, we make up the observation matrix in which each column is one surface density map for a period, and each row is a time series of observations for a given site. If a site has an unknown density value for a period, we will set it to 0 in the matrix. The arrangement of the observation matrix is arranged in zigzag.

\subsection{Method}

\subsubsection{Matrix Completion Algorithm}

In order to analyse spatial and temporal variations of density, the observation matrix is needed to be completed, which can be regarded as a special case of matrix recovery problem. If a number of known entries are given, it is possible to recover the missing entries of matrix (Chen et al., 2010). The main solution strategy of this problem is based on the minimization of the nuclear norm, which requires singular value decomposition. In many applications, it is reasonable to assume that the matrix to be recovered is low-rank (Chen et al., 2010). In this paper, we make use of three methods to recovery observation matrix, they are LMaFit (Wen et al., 2012), IALM (Chen et al., 2010) and FPCA (Ma et al., 2011), respectively. Each method is run for several times to take out the result with the highest sampling rate and the minimum error of mean square. At the end, there may be very little data in the matrix that is not recovered, in this case we will use the average of density observations for a site to represent the missing density of a period. Prior to matrix completion, retain some known data for cross-validation to ensure the accuracy of matrix completion, and the result of cross-validation will be shown later.

\subsubsection{Empirical Orthogonal Function}

EOF is an effective method for analysing the spatial and temporal variations of the geophysical field (Björnsson et al., 1997). It identifies linear transformations of the geophysical field and concentrates as much variance as possible to a few variables (Preisendorfer et al., 1988) and has been widely used in oceanographic and meteorological analyses. After matrix completion, a full surface snow density matrix can be got with the time series of surface snow density at each observation site. In this study, we adopt EOF method to decompose the observation matrix and only take first several lower-order modes, because these modes already contain most information of the observation matrix. Finally, a map of surface snow 
density covering about $97.9 \%$ of the East Antarctic area can be generated by multiplying the modes interpolated by kriging and the corresponding amplitude of the modes.

\section{RESULT AND DISCUSSION}

\subsection{Results}

\subsubsection{Pre-processing Results}

In order to ensure that densities come from same depth, we processed the data and get the mean densities from the surface to $2 \mathrm{~m}$ depth. But there are still a few sites cannot obtain the average density value, we took the density at a particular depth within 0 to $2 \mathrm{~m}$. A total of 10 observations were removed and 2229 observations were remained as shown in Fig. 2.

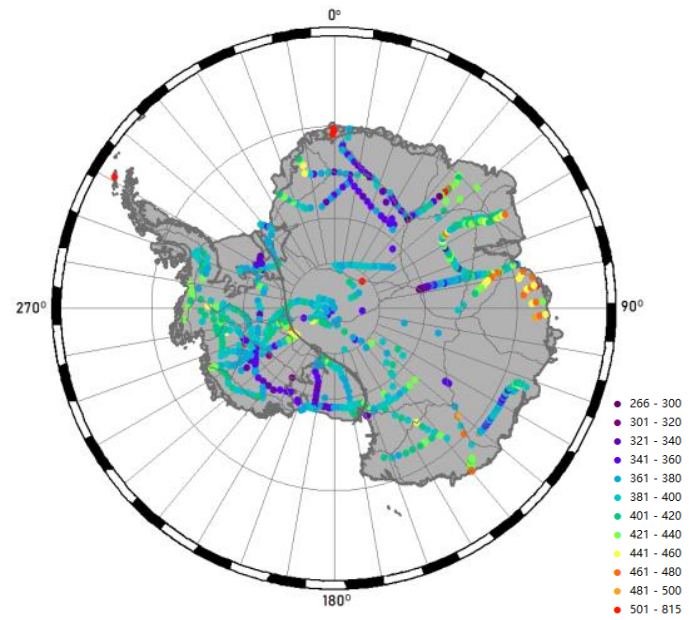

Figure 2. Locations and densities of 2229 observations after pre-processing

\subsubsection{Cross Validation Results}

In the initial experiment, we separately produced the nearsurface density maps of the East and West Antarctica since obtaining time for in-situ observations are quite different for them. For East Antarctica, we retained 7 observations, which are almost evenly distributed in space and time as shown in Fig. 3 , for cross-validation to evaluate the accuracy of matrix completion.

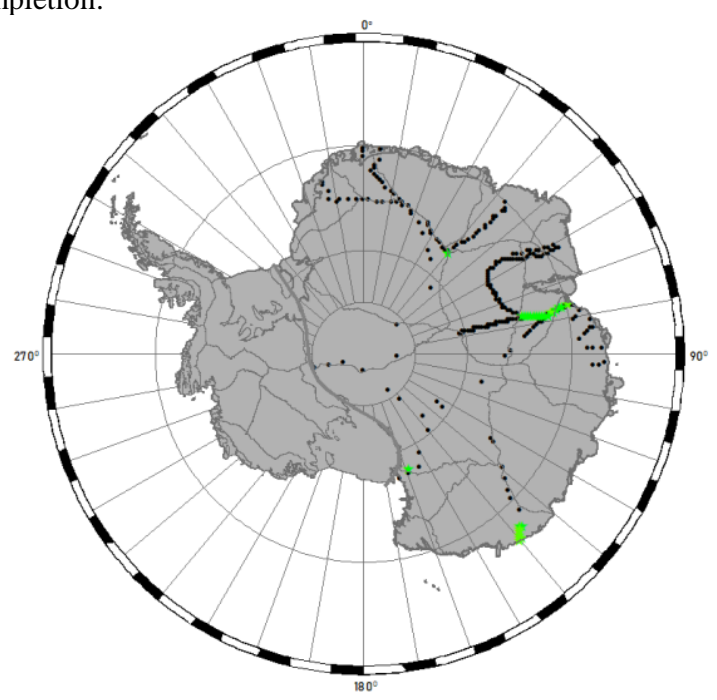

Figure 3. The density observations (black dots) at East Antarctica and green stars are the observation sites that can be used for cross-validation.
The cross-validation result is the mean difference between density observation and recovered data is $33.4 \mathrm{~kg} / \mathrm{m}^{3}$ with root mean square error about $41.2 \mathrm{~kg} / \mathrm{m}^{3}$.

\subsubsection{A Map of Surface Density}

The preliminary result of East Antarctic near-surface snow density map is shown in Figure 4. It presents the average surface density from 1991 to 2015 .

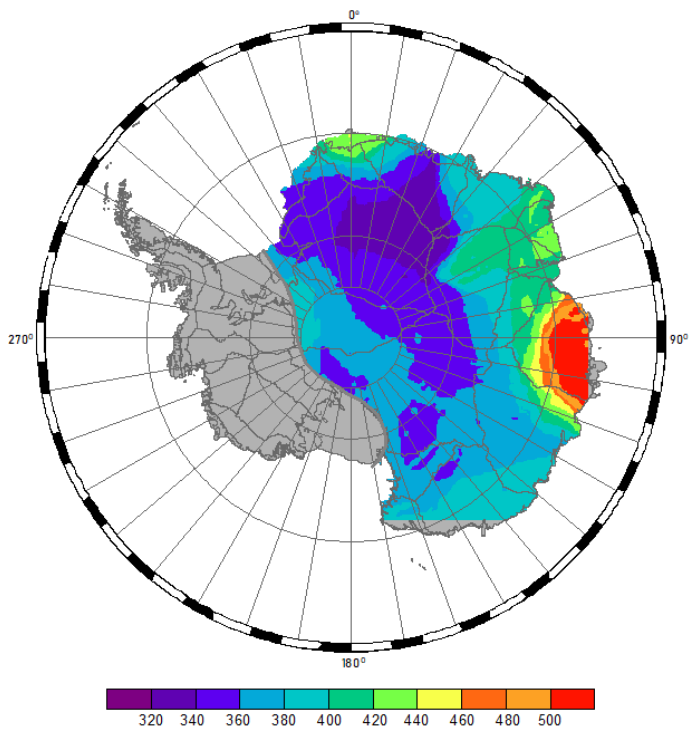

Figure 4. East Antarctic near-surface snow density map

\subsection{Comparative Analysis}

We compared the difference between our East Antarctic density map and the modelled density map, that is calculated according to the method described by Ligtenberg et al. (2011) at the same time period. Modelled result is shown in Figure 5(a) and the differences between our result and model is shown in Figure 5 (b). The difference, from $122 \mathrm{~kg} / \mathrm{m}^{3}$ to $-138 \mathrm{~kg} / \mathrm{m}^{3}$, between our result and the model is obviously. Big differences mainly locate in coastal area due to less observations but huge density variation around coast.

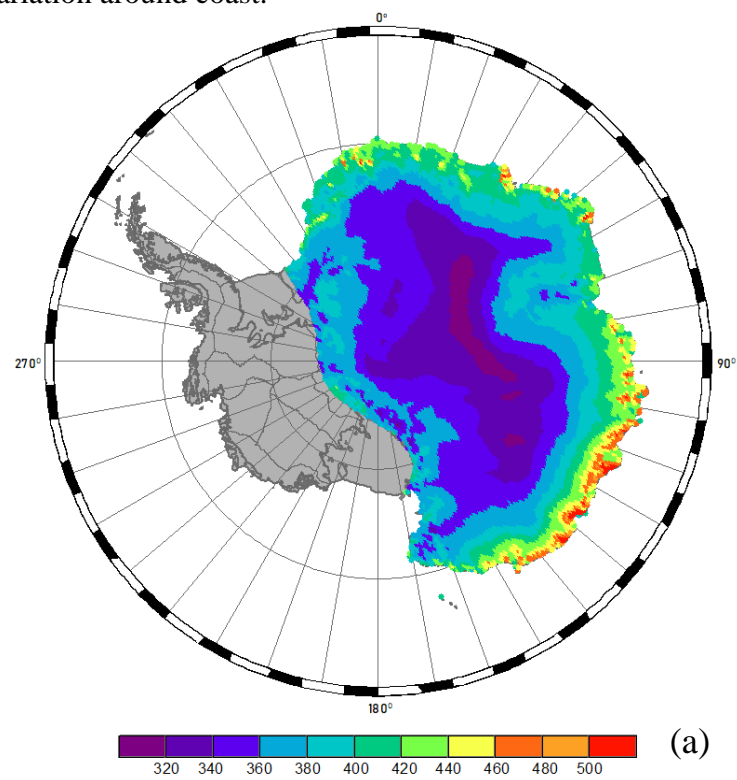




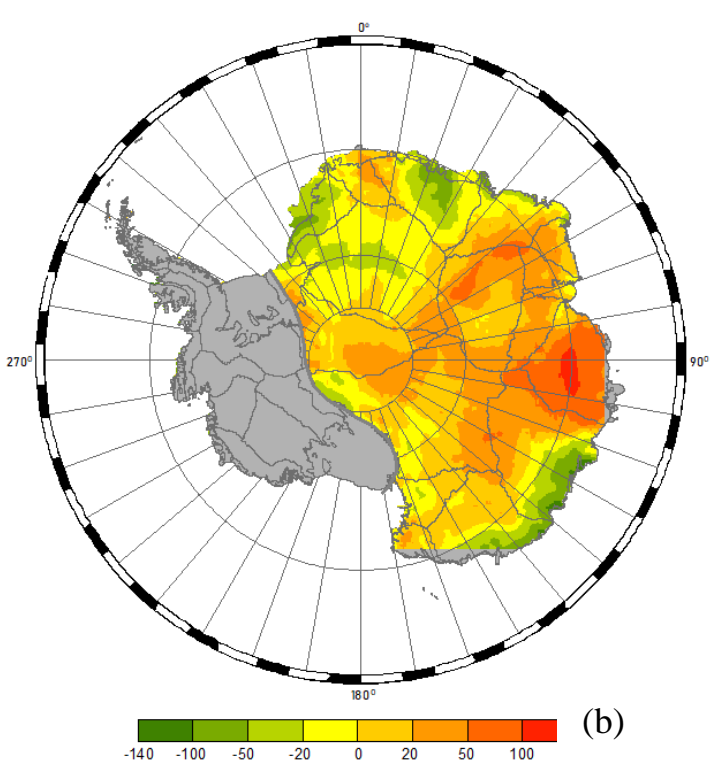

Figure 5. (a) Average modelled surface density from 1991 to 2015. (b) Difference between our result and model.

\section{SUMMARY AND CONCLUSIONS}

This paper provides new ways for the study of Antarctic surface density. A near-surface density map of East Antarctica is completely generated from in-situ density observations. We believe that the density information carried by the reliable insitu observations is more credible than empirical models and parameters. The result of density model is different from our result and big differences are concentrated on the coastal region. The largest difference is $-138 \mathrm{~kg} / \mathrm{m}^{3}$. Since there are very little observations collected from the coast, signals with fast changes in the density of the coast have not been extracted. Our results within the East Antarctica coincided well with the modelled result, with differences between -20 and 50 accounting for $77.7 \%$ of all differences. Afterwards, we will decompose the complementary observation matrix spanning from 1957 to 2015 by EOF to obtain a near-surface density map of entire Antarctica. It should be noted that the above summery is based on the preliminary data processing and analysis results.

\section{ACKNOWLEDGEMENTS}

The annual average temperature, annual average accumulation rate, and annual average wind speed required by the model are all kindly provided by Van den Broeke. The research work described in this paper has been supported by the National Key Research and Development Program of China (Project Nos. 2017YFA0603103, 2017YFA0603102 and 2017YFB0503500), the National Natural Science Foundation of China (Project Nos. 41730102 and 41571407), and the Fundamental Research Funds for the Central Universities.

\section{REFERENCES}

Arthern R J, Vaughan D G, Rankin A M, et al., 2010. In situ measurements of Antarctic snow compaction compared with predictions of models. Journal of Geophysical Research Earth Surface, 115(F3).
Björnsson, H., and Venegas, S. A., 1997. A manual for EOF and SVD analysis of climatic data, McGill University, Montreal, 52 pp.

Chen M, Lin Z, Ma Y, et al., 2010. The Augmented Lagrange Multiplier Method for Exact Recovery of Corrupted Low-Rank Matrices. Eprint Arxiv, 9.

Gunter B, Urban T, Riva R, et al., 2009. A comparison of coincident GRACE and ICESat data over Antarctica. Journal of Geodesy, 83(11):1051-1060.

Hawley R L, Brandt O, Morris E M, et al., 2008. Instruments and Methods Techniques for measuring high-resolution firn density profiles: case study from Kongsvegen, Svalbard. Journal of Glaciology, 54(186):463-468.

Ligtenberg, S. R. M, Helsen, M. M, Broeke, M. R. van den., 2011. An improved semi-empirical model for the densification of Antarctic firn. Cryosphere, 5(4):809-819.

Ma S, Goldfarb D, Chen L., 2011. Fixed point and Bregman iterative methods for matrix rank minimization. Mathematical Programming, 128(1-2):321-353.

Morris E M., 2008. A theoretical analysis of the neutron scattering method of measuring snow and ice density. Journal of Geophysical Research Earth Surface, 113(F4).

Preisendorfer R W, Mobley C D., 1998. Principal component analysis in meteorology and oceanography. Developments in Atmospheric Science, 17(50):55-72.

Rignot E., Velicogna I., van den Broeke M.R., Monaghan A., Lenaerts J., 2011. Acceleration of the contribution of the Greenland and Antarctic ice sheets to sea level rise. Geophysical Research Letters, 38, pp. L05503.

Shepherd A, Ivins E R, A G, et al., 2012. A reconciled estimate of ice-sheet mass balance. Science, 338(6111):1183.

Sugiyama S, Enomoto H, Fujita S, et al., 2012. Snow density along the route traversed by the Japanese-Swedish Antarctic Expedition 2007/08. Journal of Glaciology, 58(209):529-539.

Takahashi S, Kameda T., 2007. Instruments and Methods Snow density for measuring surface mass balance using the stake method. Journal of Glaciology, 53(183):677-680.

Wen Z, Yin W, Zhang Y., 2012. Solving a low-rank factorization model for matrix completion by a nonlinear successive overrelaxation algorithm. Mathematical Programming Computation, 4(4):333-361.

Zwally, H.J., Li J., Robbins J.W., Saba J.L., Yi D., Brenner A.C., 2015. Mass gains of the Antarctic ice sheet exceed losses, Journal of Glaciology, 61(230), 1019-1036. 